\title{
Mineração
}

\section{Modelamento matemático de peneiramento vibratório (Parte 2): simulação}

\author{
Simão Célio de Carvalho \\ Engenheiro de Minas, Sempre Viva Mineração - Construções e Transportes \\ Mestrado em Engenharia Mineral - DEMIN/UFOP \\ E-mail:simao@sviva.com.br \\ José Aurélio Medeiros da Luz \\ Universidade Federal de Ouro Preto - UFOP \\ Escola de Minas - Departamento de Engenharia de Minas - DEMIN \\ E-mail:jaurelio@demin.ufop.br
}

\section{Resumo}

O peneiramento é uma operação unitária em que mecanismos probabilísticos ocorrem de maneira intensa. Este trabalho apresenta um modelo alternativo de peneiramento a partir da abordagem probabilística de Mogensen. Um sistema computacional (SimPeneira) foi implementado e validado experimentalmente. Tal sistema é capaz de simular cenários de peneiramento industrial e quantificar o impacto de parâmetros, tais como: abertura, comprimento da peneira, velocidade do fluxo do material, área efetiva de peneiramento, freqüência e amplitude das vibrações. O sistema também pode dimensionar peneiras, utilizando-se o método clássico de dimensionamento (com modificações).

Palavras-chave: simulação, granulometria, peneiramento.

\begin{abstract}
Screening is a unit operation where probabilistic mechanisms take place in an intensive way. This work presents an alternative screening model. The final product is a software system called SimPeneira (experimentally validated) developed in order to simulate scenarios in industrial screening and to quantify the impact of parameters such as: screen opening, length, effective area, material flow speed, vibration frequency and amplitude. The system can also size screens using the classical sizing procedure (with modifications).
\end{abstract}

Keywords: simulation, size particle, screening. 


\section{Introdução}

Há tempos, os homens utilizam o processo de peneiramento com o intuito de separar produtos em diferentes granulações, formatos, bem como retirar impurezas, adequando produtos a especificações.

O peneiramento é um processo muito importante no tratamento de minérios e na separação de grãos de cereais de uma forma geral, no entanto, atualmente, são poucos os trabalhos relacionados a um processo tão utilizado em todo o mundo.

Com o intuito de simular e dimensionar o processo de peneiramento, foi desenvolvido um modelo computacional para simulação de peneiramento (sistema SimPeneira), o qual é capaz de simular diversos cenários de peneiramento, bem como dimensionar peneiras segundo o procedimento clássico, com base no método Allis Chalmers modificado (Luz \& Carvalho, 2004). Para validação do módulo de simulação anteriormente descrito, foi realizada uma série de ensaios de peneiramento em escalapiloto.

A simulação da eficiência de peneiramento e das análises granulométricas dos produtos é sempre desejada, pois, conhecendo as distribuições granulométricas dos produtos, pode-se analisar qual situação de operação dará maior eficiência operacional, sem gasto de tempo e dinheiro com amostragem e análises.

O objetivo desse trabalho é discutir o módulo de simulação de peneiras de um modelo computacional para simulação e dimensionamento de peneiramento (sistema SimPeneira), o qual é a continuação do artigo "Modelamento matemático de peneiramento vibratório (Parte 1): dimensionamento”, e, também, apresentar os resultados de algumas simulações realizadas em escala-piloto para validação do programa.

\section{Materiais e métodos}

O recurso material necessário ao desenvolvimento e validação do módu- lo de simulação do sistema computacional consistiu em microcomputador, ambiente integrado de desenvolvimento Delphi (versão 7), gerenciador de banco de dados Microsoft Access, peneira em escala-piloto localizada no CEFETOP (Centro Federal de Educação Tecnológica de Ouro Preto), britador de mandíbulas em escala-piloto, britador de rolos em escala-piloto, balança, peneirador (tipo Ro-tap), para realização das análises granulométricas, e série ABNT de peneiras. A amostra utilizada em todos os ensaios foi um material dolomítico, proveniente da Mineração Bemil Ltda., bitolado entre $9,2 \mathrm{~mm}$ e $0,3 \mathrm{~mm}$.

\subsection{Desenvolvimento do modelo proposto (SimPeneira)}

O sistema computacional SimPeneira foi desenvolvido de modo a possibilitar a simulação de diversos cenários operacionais relativos a um peneiramento em escala industrial e piloto, como, por exemplo, a abertura da peneira, a velocidade do fluxo do material, comprimento da peneira, inclinação da peneira. Ele é constituído de dois subsistemas que são: um módulo de simulação e um módulo de dimensionamento clássico, baseado no método Allis Chalmers modificado, o qual foi apresentado no artigo citado anteriormente. Essa metodologia clássica apresenta uma série de simplificações, pois não leva em conta a inclinação da peneira, a freqüência de vibração, parâmetros importantes em um processo de peneiramento. Para fazer face a esses problemas, o dimensionamento pode ser efetuado indiretamente pelo uso do módulo de simulação de peneiramento vibratório.

\subsection{Formalismo matemático do modelo de simulação}

A espessura efetiva média do peneiramento é calculada a partir da equação de continuidade, a qual, explicitada, resulta:

$$
\mathrm{E}_{\mathrm{l}}=\left(\frac{Q_{m}}{\rho_{a p} \times v \times L}\right)
$$

Onde:

$\boldsymbol{Q}_{\boldsymbol{m}}$ - vazão mássica do fluxo retido [kg/s].

$\boldsymbol{\rho}_{a p}$ - massa específica do material $\left[\mathrm{kg} / \mathrm{m}^{3}\right]$.

$\mathbf{v}$ - velocidade média de progresso do material sobre a tela [m/s].

L - largura efetiva da peneira [m].

$\mathbf{E}_{1}$ - espessura média do leito [m].

É mais conveniente, para propósitos de modelagem matemática, em vez da espessura do leito, a adoção de uma variável adimensional a ela relacionada, ou seja: uma espessura relativa. A espessura relativa aqui adotada é dada por:

$$
\mathrm{E}^{*}=\frac{\mathrm{E}_{1}}{\mathrm{a}}
$$

Onde o parâmetro $\boldsymbol{a}$ é a abertura equivalente da peneira, em metro.

Para calcular a probabilidade de uma partícula passar pela tela de uma peneira de malha quadrada, utiliza-se a expressão analítica de Mogensen (Mogensen, 1965), a qual é:

$$
p_{1}=\frac{\left[a-\phi \times f\left(\frac{d}{a}\right)-d\right] \times\left[(a+\phi) \times \cos \alpha-\left(1-f\left(\frac{d}{a}\right)\right) \times \phi-d\right]}{(a+\phi) \times[(a+\phi) \times \cos \alpha]}
$$


Onde:

a - abertura [m].

d - diâmetro da partícula [m].

$\phi$ - diâmetro do fio da tela [m].

$\boldsymbol{\alpha}$ - ângulo de inclinação da peneira plana $\left[^{\circ}\right]$.

$f\left(\frac{d}{a}\right)$ é uma função de ajuste, cujo valor (nos limites $0 \leq d / a \geq 1$ ) pode ser estimado a partir da equação seguinte, obtida por análise de regressão, a partir dos dados de Mogensen (Mogensen, 1965):

$$
f\left(\frac{d}{a}\right)=-0,27 \times \frac{d}{a}+0,27
$$

Naturalmente, se o tamanho relativo (d/a) for maior que 1,0, a função $f\left(\frac{d}{a}\right)=0,0$. A correlação estatística para a regressão da equação anterior foi de $97,96 \%$.

A probabilidade, $\boldsymbol{p}_{n}$, de uma partícula isolada da classe de tamanhos $\boldsymbol{i}$ passar pela peneira somente na n-ésima tentativa de passagem (apresentação) é dada pela equação:

$p_{n}=1-\left(1-p_{1}\right)^{n}$

Onde:

$\boldsymbol{p}_{1}$ - probabilidade de passagem de uma partícula da classe $\boldsymbol{i}$ em uma única apresentação à tela [-].

$\boldsymbol{n}$ - número de apresentações (tentativas) de passagem [-].

O número de apresentações é monotonicamente crescente com o tempo de residência sobre a tela. Por seu turno, o tempo de residência é dado por:

$$
\tau=\frac{C}{v}
$$

Onde:

C - comprimento da peneira [m].

$\boldsymbol{v}$ - velocidade média de fluxo [m/s].

O número de apresentações de cada partícula de subtamanho (undersize) às aberturas da tela é, em geral, apenas uma fração do número de ciclos da peneira, durante o tempo de residência, por causa das interações entre as partículas no leito. No presente modelo, a chamada efetividade representa a razão entre o número de ciclos durante o percurso sobre a tela e o número efetivo de apresentações.

$y=\frac{n}{f \times \tau}$

Naturalmente, no algoritmo, é necessário o conhecimento, ou da efetividade ou, alternativamente, da granulometria do material passante. No segundo caso, o sistema irá ajustar a efetividade (etapa de calibração). Assim, o número de apresentações de cada partícula à tela pode ser calculado com base no tempo de residência, $\tau$, considerando-se uma "efetividade $(\boldsymbol{y})$ " da freqüência operacional, conforme o disposto na equação a seguir:

$$
n=f \times \tau \times y
$$

Onde:

$\boldsymbol{y}$ - efetividade [-].

$f$ - freqüência $[\mathrm{Hz}]$.

$\tau$ - tempo de residência [s].

É natural supor que a dificuldade de migração dos subtamanhos dentro do leito, em direção à superfície de separação (tela), seja crescente com a espessura relativa do leito. Assim, pode-se adotar:

$y=\frac{k}{\left(E^{*}\right)^{z}}$

Onde:

$\boldsymbol{k}$ - coeficiente de efetividade [-].

$\mathbf{z}$ - ponderador exponencial de espessura [-].

Para efeito de simplificação do modelo, adotou-se, nesse trabalho, o valor: $\mathbf{z}=1,0$. Como conseqüência, o erro associado a tal adoção, na realidade, fica incorporado pela efetividade $(\boldsymbol{y})$.

Outra simplificação do presente modelo é o fato de não se levar em conta o efeito da estratificação do leito durante o processo de peneiramento (incluindo-se a estratificação grânulo-densitária do leito, similar à da jigagem).
Para o caso de o coeficiente de efetividade estar sendo inferido por valores experimentais da partição, isto é, durante a fase de calibração do modelo, o sistema utiliza uma rotina simplificada de minimização da soma dos erros quadráticos.

Em suma, a determinação de $\boldsymbol{y}$, para dado caso operacional, é o ponto chave para o modelamento do processo, no algoritmo adotado pelo SimPeneira.

O sistema calcula também as vazões mássicas da alimentação, do retido e do passante:

$Q_{m}=\frac{\sum m}{\tau_{a} \times F_{f}}$

Onde:

$\boldsymbol{\Sigma} \boldsymbol{m}$ - somatório das massas retidas em cada faixa granulométrica.

$\boldsymbol{F}_{\boldsymbol{f}}$ - fração de fluxo amostrado.

$\tau_{a}$ - tempo amostral [s].

A área efetiva de peneiramento é dada por:

$A=C \times L$

Onde:

C - comprimento da peneira (dado de entrada) [m].

L - largura da peneira [m].

A eficiência de remoção de finos pode ser calculada pela expressão convencional:

$\varepsilon=\frac{m_{u}}{m_{a}}$

Onde:

$\boldsymbol{m}_{\boldsymbol{u}}$ - massa (ou vazão)de subtamanho passante [kg].

$\boldsymbol{m}_{\boldsymbol{a}}$ - massa (ou vazão)de subtamanho alimentada [kg].

\section{Resultados e discussão}

O Programa SimPeneira, como dito anteriormente, é um software desenvol- 
vido para o dimensionamento e simulação de peneiras em escala industrial e piloto, a interface do módulo de simulação pode ser observada na Figura 1.

Ela apresenta a interface do módulo de simulação, com a rotina de ajuste do coeficiente de efetividade selecionada. Nesse caso, torna-se necessário entrar com os dados práticos de um ensaio de peneiramento. No exemplo, entramos com os dados de um dos ensaios realizados.

Na Tabela 1 e nas Figuras 2, 3 e 4, está apresentado um resumo dos resultados de ensaios realizados e simulados em uma peneira vibratória em escala-piloto. O efeito da variação da abertura da tela pode ser observado nas curvas das Figuras 2, 3 e 4.

Pode-se observar também que, quanto maior a inclinação, menor a espessura relativa do leito como seria de se esperar. Analisando-se os dados, pode-se perceber também que uma maior abertura de tela apresenta uma tendência de espessura relativa maior.

Não se conseguiu extrair uma regra ou equação que descrevesse, com rigor quantitativo, o comportamento do coeficiente de efetividade, indicando a necessidade de estudos suplementares, com possível introdução de parâmetros não considerados no modelo.

\section{Conclusões}

O modelo computacional foi concebido primacialmente para simulação

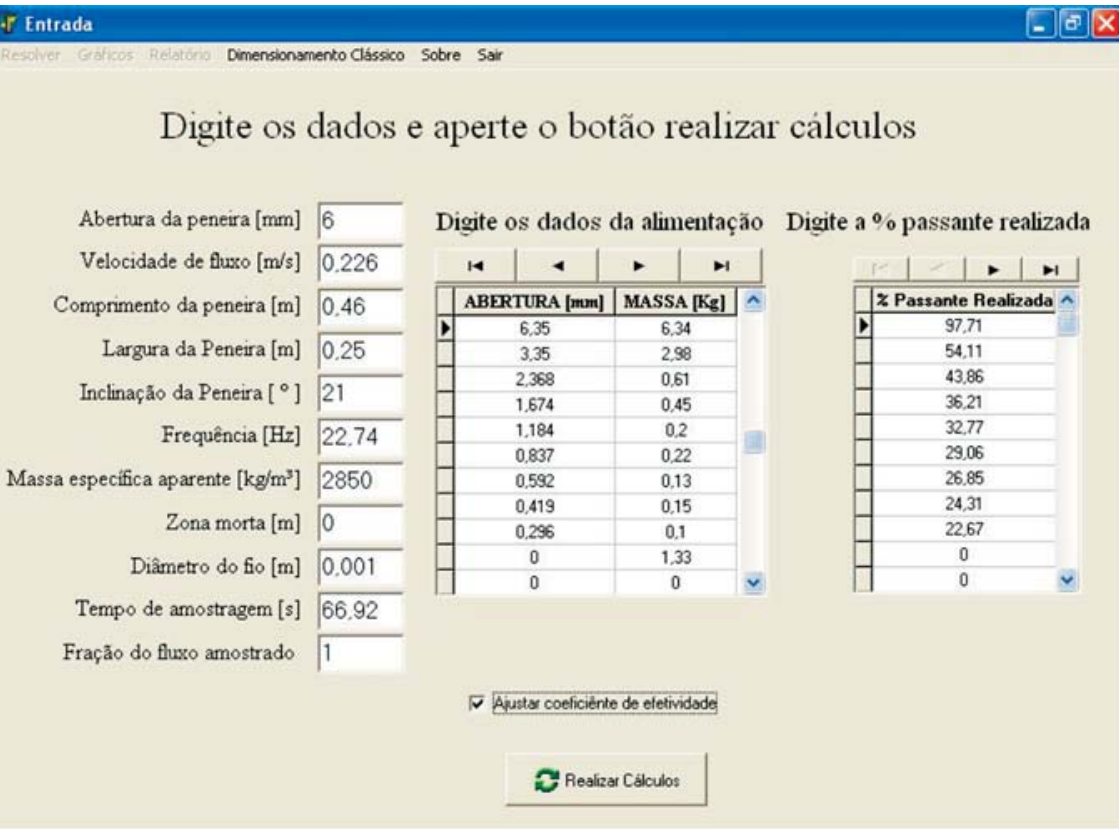

Figura 1 - Módulo de simulação do programa SimPeneira, entrando-se com o Análise Granulométrica do material passante (para calibração do modelo).

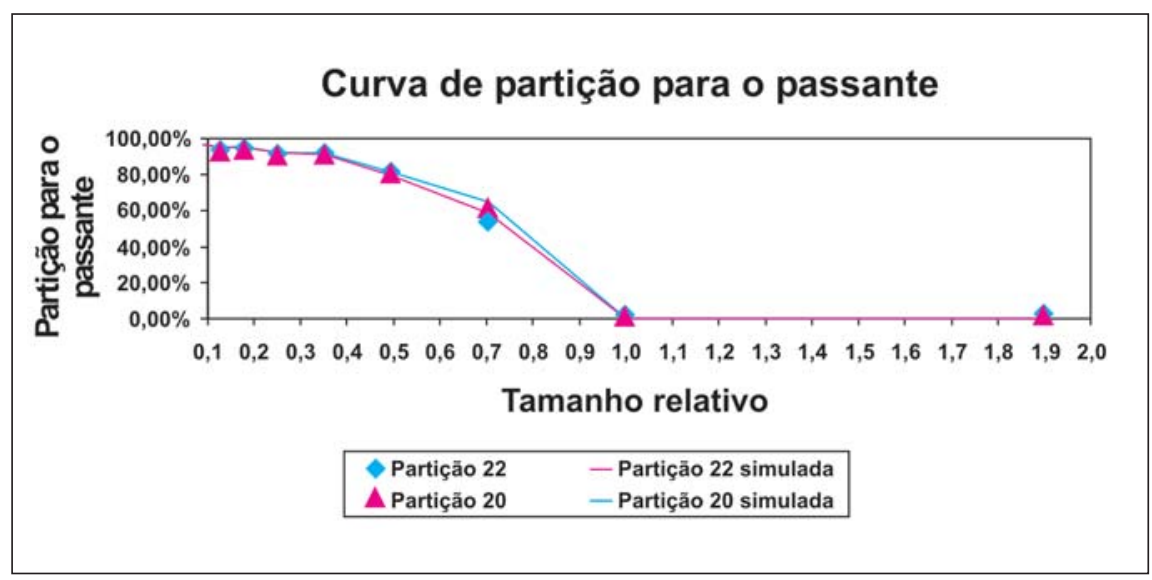

Figura 2 - Curvas de partição "Realizadas x Simuladas" (ensaios 20 e 22).

Tabela 1 - Quadro-resumo, comparando-se alguns dos resultados simulados.

\begin{tabular}{|c|c|c|c|c|c|c|}
\hline $\begin{array}{l}\text { Coeficiente de } \\
\text { efetividade } k \text { [-] }\end{array}$ & $\begin{array}{l}\text { Espessura do } \\
\text { leito } E \text { [mm] }\end{array}$ & $\begin{array}{l}\text { Espessura } \\
\text { relativa } E^{\star}[-]\end{array}$ & $\begin{array}{c}\text { Abertura a } \\
{[\mathrm{mm}]}\end{array}$ & Ensaio & $\begin{array}{l}\text { Vazão } \\
{[\mathrm{kg} / \mathrm{s}]}\end{array}$ & $\begin{array}{c}\text { Inclinação da } \\
\text { peneira }\left[{ }^{\circ}\right]\end{array}$ \\
\hline 0,10 & 0,009 & 2,90 & 3,35 & 20 & 0,34 & 11 \\
\hline 0,08 & 0,005 & 1,59 & 3,35 & 22 & 0,33 & 16 \\
\hline 0,60 & 0,004 & 0,66 & 6,00 & 27 & 0,26 & 16 \\
\hline 0,67 & 0,008 & 1,27 & 6,00 & 30 & 0,34 & 11 \\
\hline 0,63 & 0,002 & 2,44 & 0,83 & 35 & 0,26 & 21 \\
\hline 0,37 & 0,001 & 1,70 & 0,83 & 36 & 0,34 & 21 \\
\hline
\end{tabular}




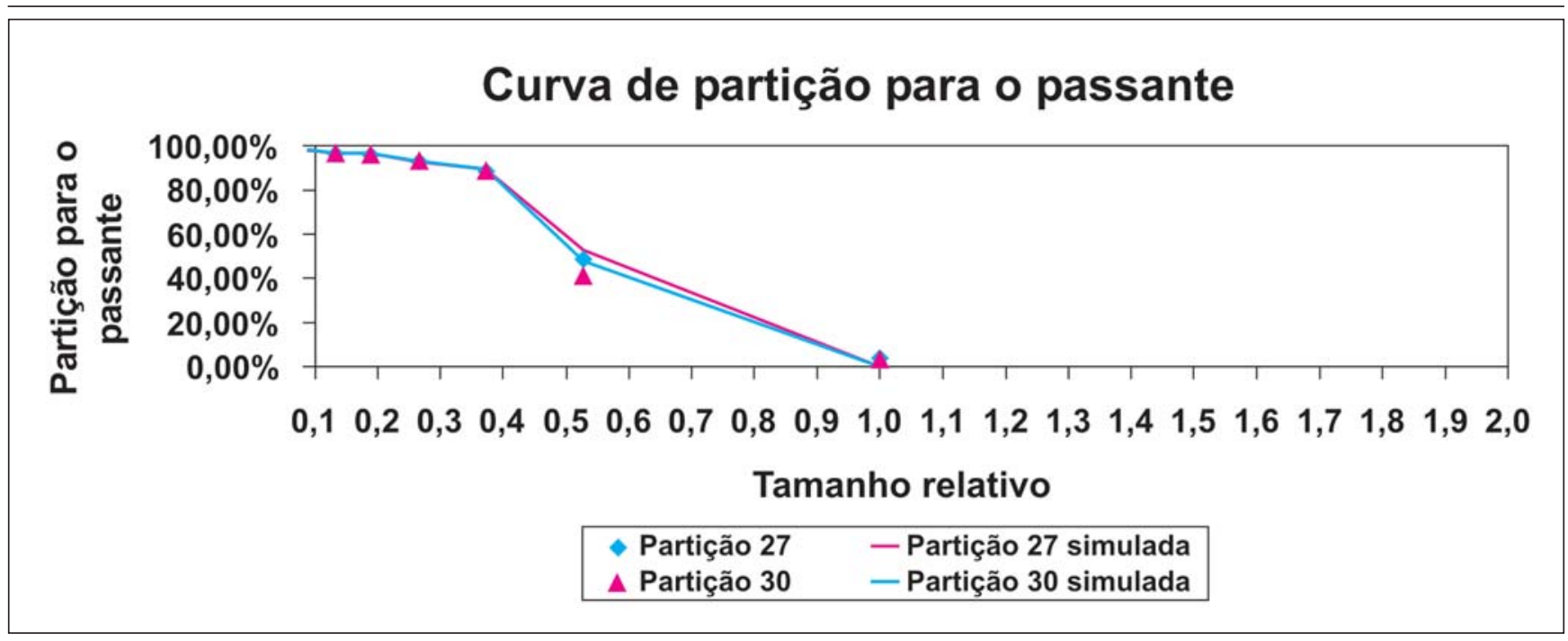

Figura 3 - Curvas de partição "Realizadas x Simuladas" (Ensaios 27 e 30).

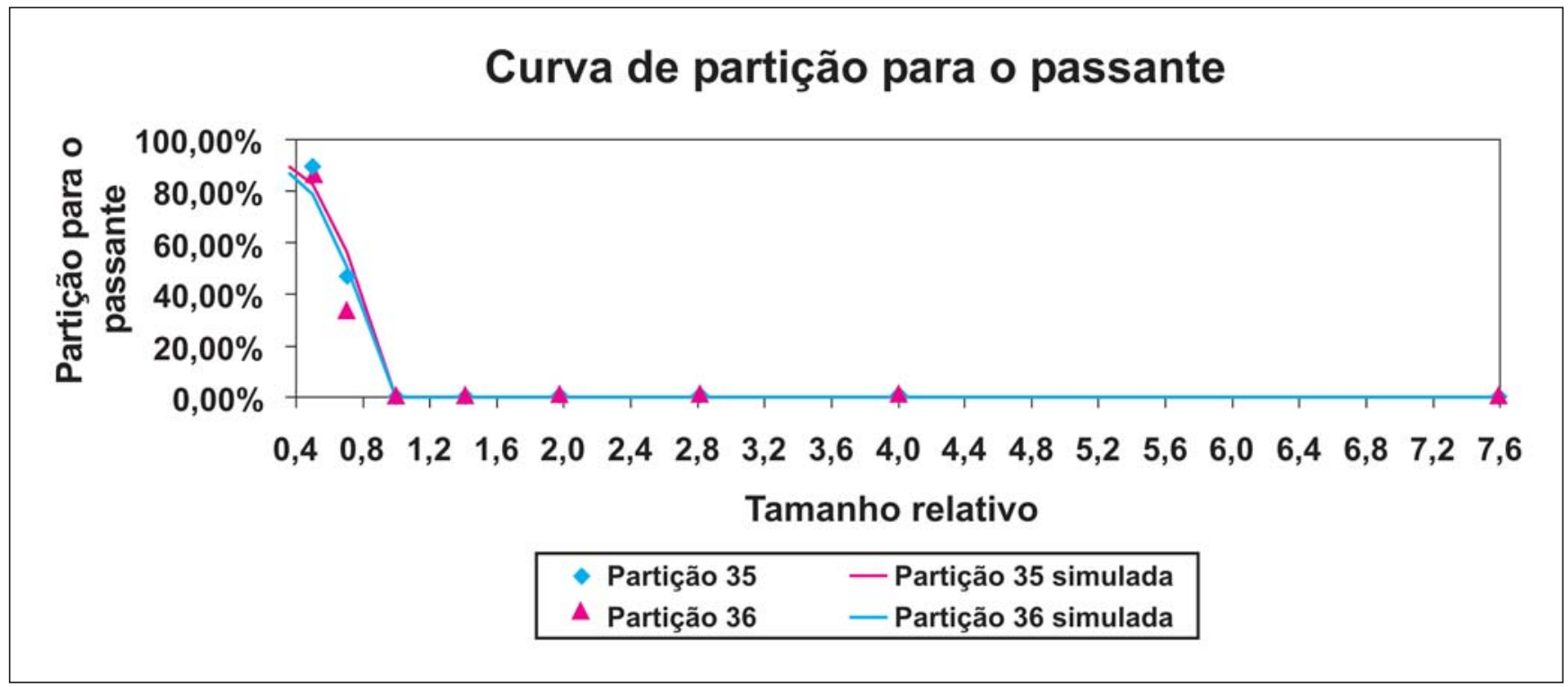

Figura 4 - Curvas de partição "Realizadas x Simuladas" (Ensaios 35 e 36).

probabilística de peneiramento vibratório (sistema SimPeneira), partindo-se do formalismo de Mogensen (Mogensen 1965, Kelly \& Spottiswood, 1982). Esse modelo possibilita a simulação de diversos cenários de um peneiramento em escala industrial e piloto. Os resultados mostram uma boa aderência entre os dados experimentais e os simulados.

Tal aderência estatística foi obtida pela convergência de valor do coeficiente de efetividade para várias condições experimentais. Essa é uma grande vantagem do modelo proposto, em relação a outros, pois ele requer a calibração de apenas um parâmetro, o coeficiente de efetividade.

Ressalta-se que o programa também pode servir como excelente suporte didático nos cursos regulares de engenharias de minas, metalúrgica e química.

\section{Referências bibliográficas}

CHAVES, A. P., PERES, A. E. C. Teoria e prática do tratamento de minérios britagem, peneiramento e moagem. 1. ed. São Paulo: Signus, 1999. v. 3. 237p.
FAÇO. Manual de britagem da Faço. São Paulo: Allis Mineral Systems, 1994.

KELLY, E. G., SPOTTISWOOD, D. J. Introduction to mineral processing. New York: John Wiley, 1982.

LUZ, J. A. M., CARVALHO, S.C. Modelamento matemático de peneiramento vibratório (parte 1): dimensionamento clássico. REM Revista Escola de Minas. Ouro Preto: 2004 [no prelo].

MOGENSEN, F. A new screening method of screening granular materials. The Quarry Managers' Journal. p. 409-414. Oct., 1965.

Artigo recebido em 16/11/2004 e aprovado em 03/01/2005. 